\title{
Polyhedral results for the Cardinality Constrained Multi-cycle Problem (CCMcP) and the Cardinality Constrained Cycles and Chains Problem (CCCCP)
}

\author{
$\underline{\text { V. Mak-Hau }}^{\text {a }}$ \\ ${ }^{a}$ School of Information Technology, Deakin University, Australia \\ Email: vicky.mak@deakin.edu.au
}

\begin{abstract}
In recent years, there has been studies on the cardinality constrained multi-cycle problems on directed graphs, some of which considered chains co-existing on the same digraph whilst others did not. These studies were inspired by the optimal matching of kidneys known as the Kidney Exchange Problem (KEP). In a KEP, a vertex on the digraph represents a donor-patient pair who are related, though the kidney of the donor is incompatible to the patient. When there are multiple such incompatible pairs in the kidney exchange pool, the kidney of the donor of one incompatible pair may in fact be compatible to the patient of another incompatible pair. If Donor A's kidney is suitable for Patient B, and vice versa, then there will be arcs in both directions between Vertex A to Vertex B. Such exchanges form a 2-cycle. There may also be cycles involving 3 or more vertices. As all exchanges in a kidney exchange cycle must take place simultaneously, (otherwise a donor can drop out from the program once his/her partner has received a kidney from another donor), due to logistic and human resource reasons, only a limited number of kidney exchanges can occur simultaneously, hence the cardinality of these cycles are constrained. In recent years, kidney exchange programs around the world have altruistic donors in the pool. A sequence of exchanges that starts from an altruistic donor forms a chain instead of a cycle. We therefore have two underlying combinatorial optimization problems: Cardinality Constrained Multi-cycle Problem (CCMcP) and the Cardinality Constrained Cycles and Chains Problem (CCCCP). The objective of the KEP is either to maximize the number of kidney matches, or to maximize a certain weighted function of kidney matches.
\end{abstract}

In a CCMcP, a vertex can be in at most one cycle whereas in a CCCCP, a vertex can be part of (but in no more than) a cycle or a chain. The cardinality of the cycles are constrained in all studies. The cardinality of the chains, however, are considered unconstrained in some studies, constrained but larger than that of cycles, or the same as that of cycles in others. Although the CCMcP has some similarities to the ATSP- and VRPfamily of problems, there is a major difference: strong subtour elimination constraints are mostly invalid for the CCMcP, as we do allow smaller subtours as long as they do not exceed the size limit. The CCCCP has its distinctive feature that allows chains as well as cycles on the same directed graph. Hence, both the CCMcP and the CCCCP are interesting and challenging combinatorial optimization problems in their own rights.

Most existing studies focused on solution methodologies, and as far as we aware, there is no polyhedral studies so far. In this paper, we will study the polyhedral structure of the natural arc-based integer programming models of the CCMcP and the CCCCP, both containing exponentially many constraints. We do so to pave the way for studying strong valid cuts we have found that can be applied in a Lagrangean relaxation-based branch-and-bound framework where at each node of the branch-and-bound tree, we may be able to obtain a relaxation that can be solved in polynomial time, with strong valid cuts dualized into the objective function and the dual multipliers optimised by subgradient optimisation.

Keywords: Integer programming, combinatorial optimization, cardinality constrained cycle problems, kidney exchange, clearing barter exchange 


\section{INTRODUCTION}

In this paper, we will present arc-based integer programming models of the Cardinality Constrained Multicycle Problem $(\mathrm{CCMcP})$ and the Cardinality Constrained Cycles and Chains Problem (CCCCP) and study their polyhedral structures, in the hope of finding strong constraints to be applied in a branch-and-bound framework for finding exact solutions in computationally efficient ways. Preliminary results of the exponential-size arc based formulations for the CCMcP and the CCCCP are presented in a companion paper Mak-Hau (2015a).

\section{The Cardinality Constrained Multi-cycle Problem}

\subsection{Related problems}

The Cardinality Constrained Multi-cycle Problem (CCMcP) arose from the Kidney Exchange Problem (KEP). A detailed description of the various forms of the KEP can be found in Mak-Hau (2015a). We first describe the mathematical problem. Let $D=(V, A)$ be a directed graph, with $V$ the set of vertices and $A$ the set of arcs. The Cardinality Constrained Multi-cycle Problem (CCMcP) is defined as follows. A feasible solution of the $\mathrm{CCMcP}$ may contain more than one cycle but is not required to involve all vertices on $D$. Each cycle must contain no more than $K$ vertices. There is a weight associated with each arc: we denote the weight by $w_{a}$, for all $a \in A$. The objective of the CCMcP is to maximise the sum of the weights from the arcs used in the cycles.

The CCMcP on directed graphs is closely related to a number of well-known combinatorial optimisation problems. The Cardinality Constrained Travelling Salesman Problem (Cao and Glover (1997)) and the Cardinality Constrained Knapsack Problem defined on undirected graphs (Bauer et al. (2002)) consider only single cycle, whilst the CCMcP allows multiple cycles. Other problems that allow multiple cycles, e.g., the Cardinality Constrained Covering Travelling Salesman Problem (Patterson and Rolland (2003)), require that all vertices be visited; this is not the case for the $\mathrm{CCMcP}$. Some constrained single- or multi-cycle problems require that exactly $K$ vertices must be used in a cycle, see, e.g., the $K$-cycle Problem (Nguyen and Maurras (2001) and Hartmann and Özlük (2001)). In the case of CCMcP, a feasible solution can contain cycles with less than $K$ vertices. The $\mathrm{CCMcP}$ is also different from the $k$-Cardinality Constrained Assignment Problem, (see, e.g., Bai (2009); Dell'Amico and Martello (1997); Dell'Amico et al. (2001); Volgenant (2004)) as the latter requires that the total number of vertices used in all subtours must not exceed $K$. The most closely related problem, however, is the Capacitated Vehicle Routing Problems (CVRP) on directed graphs (see, e.g., Cornuejols and Harche (1993), Toth and Vigo (2002) and Baldacci et al. (2010)) and the Asymmetric Travelling Salesman Problem with Replenishment arcs (RATSP) (see, e.g., Mak and Boland (2000, 2006, 2007)). Although, both of CVRP and the RATSP require that all vertices be visited.

\subsection{A brief review of the literature}

The CCMcP is mostly studied in the context of kidney (or barter) exchange, with Roth et al. (2007) the first to propose an arc-based and a cycle-based integer programming models. The former involves $\mathcal{O}\left(|V|^{2}\right)$ variables but exponentially many constraints, whilst the latter has exponentially many variables, but only $|V|$ constraints. Abraham et al. (2007) presented a column-generation approach for the cycle-based formulation and provided detailed description on the branch-and-price method, though only $K=3$ was considered in the implementation. Manlove and O'Malley (2012) also implemented the cycle-based formulation, but column-generation was not described. Presumably all columns are explicitly generated as only $K=3$ and $K=4$ were considered in the numerical experiments. Constantino et al. (2013) presented a polynomial-size arc-based formulation, wherein multiple copies of the digraph $D$ was made so that each cycle will appear in exactly one copy of the digraph. Symmetry elimination and problem reduction schemes were implemented. The polynomial-size model is compared against the cycle-based model (though the same problem reduction schemes could not be implemented for the latter). The cycle-based model was computationally more efficient for problems with low arc-density, for $K=3$ and $K=4$, but the polynomial-size model was better for problems with medium and high arc-density, for $K=5$ and $K=6$. No other values of $K$ was tested. Klimentova et al. (2014) proposed a decomposition method that combines column generation and the graph cloning idea of Constantino et al. (2013). The column generation problem, though presented as an integer programming problem, was solved using heuristic methods. For a detailed review of the various integer programming models, solution methods, and numerical results of previous work, see Mak-Hau (2015a). 


\subsection{The arc formulation}

There is very little work in the literature that implement the arc formulation presented in Roth et al. (2007). The formulation is given as follows. Let: $x_{i j}$, for all $(i, j) \in A$ be a binary decision variable such that $x_{i j}=1$ if $\operatorname{arc}(i, j)$ is used, and $x_{i j}=0$ otherwise; let $\pi=\left(i_{1}, \ldots, i_{K+1}\right)$ be a minimal cardinality-violation path (MVP); and let $\Pi$ be the set of all MVPs. Recall that $w_{i j}$ is the cost of arc $(i, j)$.

Model 1 (The Arc Formulation of Roth et al. (2007)).

$$
\begin{array}{lll}
\max & \sum_{(i, j) \in A} w_{i j} x_{i j} & \\
\text { s.t. } & \sum_{(i, j) \in A} x_{i j} \leq 1, \quad \forall i \in V \\
& \sum_{(j, i) \in A} x_{j i} \quad=\sum_{(i, j) \in A} x_{i j}, \quad \forall i \in V \\
& \sum_{(i, j) \in \pi} x_{i j} \leq K-1, \quad \forall \pi \in \Pi \\
& x_{i j} \in\{0,1\}, \quad \forall(i, j) \in A . &
\end{array}
$$

Constraints (2) and (3) ensure that each vertex is involved in no more than one cycle, and that the balance of flow for all vertices is observed. Constraint (4) eliminates all MVPs, and there are exponentially many of them.

\section{A POlyhedral STUdy OF THE CCMCP}

For the sake of polyhedral analysis, we consider the CCMcP defined on a complete directed graph. This assumption is not restrictive as we can assign a cost of $-\infty$ for $\operatorname{arcs}$ that do not exist. Let $\mathcal{K}_{n}=(V, A)$ be a complete digraph with $n$ vertices and no loops. We have that $|V|=n$ and $|A|=n(n-1)$.

Proposition 1 (Dimension of the CCMcP polytope). Let $\mathcal{P}_{n, K}$ be the CCmC Polytope defined on $\mathcal{K}_{n}$ with cardinality $K$. The dimension of $\mathcal{P}_{n, K}$ is:

$$
\operatorname{dim}\left(\mathcal{P}_{n, K}\right)= \begin{cases}\left(\begin{array}{l}
n \\
2
\end{array}\right), & K=2 ; \\
n^{2}-2 n+1, & 3 \leq K \leq n\end{cases}
$$

Proof. [Case $K=2$ ] First we have the solution with no cycles at all, i.e., the $\mathbf{0}$-vector. Then, similar to the arguments used in Hartmann and Özlük (2001) for the $p$-cycle polytope, for each distinct pair of vertices $i, j \in V, i \neq j$, we can construct a set of vectors $\mathcal{X}$ with each vector representing a solution with a simple 2-cycle that uses arcs $(i, j)$ and $(j, i)$, and there are $\left(\begin{array}{l}n \\ 2\end{array}\right)$ such vectors. The $\left(\begin{array}{l}n \\ 2\end{array}\right)+1$ vectors above are clearly affinely independent. Now, any feasible solution that contains more than one 2-cycles can be obtained as a linear combination of vectors in $\mathcal{X}$. Hence, $\operatorname{dim}\left(P_{n, 2}\right)=\left(\begin{array}{l}n \\ 2\end{array}\right)$.

[Case $K \geq 3$ ] There are $|A|=n(n-1)$ variables. Notice that $\operatorname{dim}\left(P_{n, K}\right) \leq n^{2}-2 n+1$, as the degree of freedom of the $|V|=n$ equality constraints is $n-1$, hence we are only required show that $\operatorname{dim}\left(P_{n, K}\right) \geq$ $n^{2}-2 n+1$. We do so by mathematical induction. Let $e_{i, j}$ be a $|A|$-dimensional incident vector with all entries equal to zero, except for the entry representing arc $(i, j)$, which has a value of one.

Trivial case, $P_{3,3}$. Let $V=\{1,2,3\}$. We have 5 affinely independent vectors $\left(\mathbf{0}, e_{1,2}+e_{2,1}, e_{1,3}+e_{3,1}\right.$, $e_{2,3}+e_{3,2}$, and $\left.e_{1,2}+e_{2,3}+e_{3,1}\right)$ that represent feasible solutions for $P_{3,3}$. Hence, $\operatorname{dim}\left(P_{3,3}\right)=4$.

Induction on $n$, with $K=3$. Assuming that $\operatorname{dim}\left(P_{\ell, 3}\right)=\ell^{2}-2 \ell+1$. To show that $\operatorname{dim}\left(P_{\ell+1,3}\right)=$ $(\ell+1)^{2}-2(\ell+1)+1$, we are required to find $2 \ell-1$ new, affinely independent feasible vectors.

Let the new vertex be $\alpha^{*}$, we have a set $(\Omega)$ that contains $\ell$ new solutions each containing a simple 2-cycle $\left(\alpha^{*}, j\right),\left(j, \alpha^{*}\right)$, for $j \in V$. Now consider the 3-cycles $\left(\alpha^{*}, i, 1, \alpha^{*}\right)$, one for each $i \in V \backslash\{1\}$. These 3-cycles are affinely independent to the solutions in $\Omega$, as all 2-cycles involving $\left(\alpha^{*}, i\right)$ has $\operatorname{arc}\left(i, \alpha^{*}\right)$ as well. There are $\ell-1$ such 3 -cycles. Therefore we have at least $2 \ell-1$ new affinely independent feasible solutions, thus $\operatorname{dim}\left(P_{n, 3}\right) \geq n^{2}-2 n+1$ and hence $\operatorname{dim}\left(P_{n, 3}\right)=n^{2}-2 n+1$. 
Induction on $K$, for $K \geq 3$, with $n \geq K$ unchanged. The trivial cases for each $n$, with $K=3$ is proved above. Now, for any $3 \leq K<K^{\prime} \leq n$, all feasible solutions with size no more than $K$ are feasible solutions when cardinality constraint is $K^{\prime}$. Hence, $\operatorname{dim}\left(P_{n, K}\right) \geq n^{2}-2 n+1$ and thus $\operatorname{dim}\left(P_{n, K}\right)=n^{2}-2 n+1$, for all $3 \leq K \leq n$.

Theorem 1. The trivial constraint $x_{a} \geq 0$, for all $a \in A$ are facet defining for $P_{n, K}$ defined on $\mathcal{K}_{n}$, for all $3 \leq K \leq n$.

Theorem 2. The Degree Constraints (2),

$$
\sum_{a \in \delta^{+}(i)} x_{a} \leq 1 \quad \forall i \in V
$$

are facet defining for $P_{n, K}$ defined on $\mathcal{K}_{n}$, for all $3 \leq K \leq n$.

See Mak-Hau (2015b) for proofs of Theorems 1 and 2.

\subsection{Strengthened minimal cardinality violation path elimination constraints}

Some strong minimal infeasible path elimination constraints has been found in the context of RATSP and ATSP/VRP with time windows, see Mak and Boland (2006); Mak and Ernst (2007) respectively, but they in general eliminate subtours as well, and is thus invalid for the CCMcP.

Definition 1. Let $K$ be the cardinality limit for the $C C M c P$, we define $\pi=\left\{i_{1}, \ldots, i_{K+1}\right\}$ to be a MVP.

We propose to strengthen Constraint (4) in the following ways. Let:

- $V(\pi)=\left\{i_{1}, \ldots, i_{K+1}\right\}$ be the set of vertices in a minimal violation path $\pi$;

$$
\begin{aligned}
& -x(\mathcal{F})=\sum_{s=1}^{K} \sum_{t=s+1}^{K+1} x_{i_{s}, i_{t}} ; \text { and } \\
& \text { - } x(\mathcal{B})=\sum_{s=3}^{K} \sum_{t=2}^{s-1} x_{i_{s}, i_{t}}
\end{aligned}
$$

Lemma 1. The constraints:

$$
\begin{aligned}
\sum_{(i, j) \in \pi} x_{i j}+x(\mathcal{B})+\sum_{j^{\prime} \notin V(\pi)} x_{i_{K}, j^{\prime}} & \leq K-1, \\
x(\mathcal{F}) & \leq K-1, \\
x(\mathcal{F})-\sum_{s=1}^{K-1} x_{i_{s}, i_{K+1}}+\sum_{j^{\prime} \notin V(\pi)} x_{i_{K}, j^{\prime}} & \leq K-1,
\end{aligned}
$$

for all minimal cardinality infeasible path $\pi \in \Pi$, are valid.

Proof. We provide for proof for (6) only, as similar arguments can be used for (7) and (8). Notice that if either or both of the arcs $\left(i_{1}, i_{2}\right)$ and $\left(i_{K}, i_{K+1}\right)$ are not used, then at most $K-1$ arcs can be used in the rest of the MVP $(\mathcal{P})$. Now, if both arcs are used, then all other arcs entering vertex $i_{2}$ and leaving vertex $i_{K}$ will disappear, hence making $\left(i_{2}, \ldots, i_{K}\right)$ a MVP with cardinality $K-2$. The same argument can be repeated until we obtain either of the following cases.

For $K$ odd: We are left with the path $\left(\frac{K+1}{2}-1, \frac{K+1}{2}, \frac{K+1}{2}+1, \frac{K+1}{2}+2\right)$ with three forward arcs, one backward arc $\left(\frac{K+1}{2}+1, \frac{K+1}{2}\right)$, and a cardinality limit of 3 . Clearly no more than two arcs can be used.

For $K$ even: A similar argument can be used. 


\section{The Cardinality Constrained Cycles and Chains Problem}

The Cardinality Constrained Cycles and Chains Problem (CCCCP) is more complicated. It arose from the variation of the KEP where there exists altruistic donors. Integer programming models are proposed in Glorie et al. (2014), Anderson et al. (2015), and Mak-Hau (2015a). The digraph $G=\left(V, A^{\prime}\right)$ is defined as follows. We have that $V=N \cup P$, with $N \cap P=\emptyset, A=\{(i, j) \mid i, j \in P\}$, and $A^{\prime}=A \cup\{(i, j) \mid i \in N, j \in P\}$. A cycle on $G$ involves only vertices in $P$. A chain on $G$, however, always begins with a vertex in $N$, travels through some vertices in $P$, and ends with a vertex in $P$. The cardinality restriction in terms of the number of arcs used in a chain is represented by $L$, which is generally considered to be not less than $K$. We use $\Delta$ to represent the set of MVP for chains. Let: $y_{i j} \in\{0,1\}$, for all $(i, j) \in A^{\prime}$, with $y_{i j}=1$ if arc $(i, j)$ is used as part of a chain, and 0 otherwise; $x_{i j} \in\{0,1\}$, for all $(i, j) \in A$, with $x_{i j}=1$ if arc $(i, j)$ is used as part of a cycle, and 0 otherwise. The IP model for the CCCCP is given as below.

\section{Model 2. The exponential-size SPLIT formulation}

$$
\begin{aligned}
& \max z=\sum_{(i, j) \in A^{\prime}} w_{i j} y_{i j}+\sum_{(i, j) \in A} w_{i j} x_{i j} \\
& \text { s.t. } \sum_{j:(i, j) \in A^{\prime}} y_{i j} \leq 1, \quad \forall i \in N \\
& \sum_{j:(i, j) \in A}\left(y_{i j}+x_{i j}\right) \leq 1, \quad \forall i \in P \\
& \sum_{j:(i, j) \in A} y_{i j} \leq \sum_{j:(j, i) \in A^{\prime}} y_{j i} \\
& \sum_{j:(i, j) \in A} x_{i j}=\sum_{j:(j, i) \in A} x_{j i} \\
& \sum_{j:(i, j) \in A} y_{i j} \leq|S|-1, \quad \forall S \subset P, 2 \leq|S| \leq|P|-2 \\
& \sum_{(i, j) \in \delta} y_{i j} \leq L-1, \quad \forall \delta \in \Delta \\
& \sum_{(i, j) \in \pi} x_{i j} \leq K-1, \quad \forall \pi \in \Pi .
\end{aligned}
$$

Notice that the number of $x$ - and $y$ - variables are $|P|(|P|-1)$ and $|N||P|+|P|(|P|-1)$ respectively. Constraints $(9)-(12)$ are the flow balance and degree constraints. Constraint $(13)$ eliminates the formation of subtours from vertices in $P$ induced by the $y$ variables, and Constraints $(14)$ and $(15)$ eliminate the formation of MVPs in chains and cycles respectively. Notice that instead of Constraints (14) for the elimination of MVPs, some strong constraints in the context of RATSP or CVRP can be implemented, given that those constraints eliminate subtours as well.

Proposition 2 (Dimension of the CCCCP polytope). Let $|N|=n,|N| \geq 2 ;|P|=p,|P| \geq 2$; and let $\mathcal{Q}_{n, p, K, L}$ be the CCCC Polytope defined on a directed graph where: (1) vertices in $P$ defines a complete graph; (2) arc ( $i, j$ ) exists for all $i \in N$ and all $j \in P$; (3) $K$ (for $K \geq 2$ ) is the cardinality restriction for cycles; and (4) $L$ (for $L \geq 2$ ) is the cardinality restriction for chains. The dimension of $\mathcal{Q}_{n, p, K, L}$, is:

$$
\operatorname{dim}\left(\mathcal{Q}_{n, p, K, L}\right)= \begin{cases}\left(\begin{array}{l}
p \\
2
\end{array}\right)+n p+p(p-1), & K=2 \\
p^{2}-2 p+1+n p+p(p-1), & 3 \leq K \leq n\end{cases}
$$

Proof. We expand the incident vectors $e_{a}$ to contain $e_{a}^{y}$ and $e_{a}^{x}$, for all $a \in A$, as well as $e_{a}^{y}$, for all $a \in A^{\prime} \backslash A$ to represent a vector of zeros except for the entry representing arc $a$ being used in a chain (with superscript $y$ ) or a cycle (with superscript $x$ ) which has a value of one. The original $\left(\begin{array}{l}p \\ 2\end{array}\right)$ two-cycles in the case of $K=2$ and the $p^{2}-2 p+1$ two- and three-cycles in the case of $K=3$, together with the null vector, are still feasible solutions for the CCCCP. We are now required to construct at least $n p+p^{2}-p$ feasible solutions that involves only chains. As there are $n$ nodes in $N$, each connecting to every vertices is $P$, we have $n p$ arcs, and the corresponding incident vectors $e_{s, i}^{y}$, for all $s \in N$ and all $i \in P$ are affinely independent. Now, we consider 
paths that begin from any of the vertices in $N$ and visit two distinct vertices in $P$, forming incident vectors $e_{s^{*}, i}^{y}+e_{i, j}^{y}$, for any arbitrary $s^{*} \in N$, and all distinct $i, j \in P$. We have $p^{2}-p$ such affinely independent incident vectors, hence the proposition is proved.

Theorem 3. Let $|N|=n,|N| \geq 2 ;|P|=p,|P| \geq 2$; and let $\mathcal{Q}_{n, p, K, L}$ be the CCCC Polytope defined on a directed graph where: (1) vertices in $P$ defines a complete graph; (2) arc ( $i, j)$ exists for all $i \in N$ and all $j \in P$; (3) $K$ (for $K \geq 2$ ) is the cardinality restriction for cycles; and (4) $L$ (for $L \geq 2$ ) is the cardinality restriction for chains. The trivial constraints $x_{a} \geq 0$, for all $a \in A$, and $y_{a} \geq 0$, for all $a \in A^{\prime}$, for $K \geq 2$ and $L \geq 2$ are facet defining for $\mathcal{Q}_{n, p, K, L}$.

We now examine the non-trivial constraints.

Theorem 4. Let $|N|=n,|P|=p$, and let $\mathcal{Q}_{n, p, K, L}$ be the CCCC Polytope defined on a directed graph where: (1) vertices in $P$ defines a complete graph; (2) arc $(i, j)$ exists for all $i \in N$ and all $j \in P$; (3) $K$ (for $K \geq 2$ ) is the cardinality restriction for cycles; and (4) $L$ (for $L \geq 2$ ) is the cardinality restriction for chains. The degree constraint (9) is facet-defining for $\mathcal{Q}_{n, p, K, L}$ under the conditions that $|N| \geq 2 ;|P| \geq 3$ for $K=2$, and $|P| \geq 4$ for $K \geq 3$.

Theorem 5. Let $|N|=n,|P|=p$, and let $\mathcal{Q}_{n, p, K, L}$ be the CCCC Polytope defined on a directed graph where: (1) vertices in $P$ defines a complete graph; (2) arc $(i, j)$ exists for all $i \in N$ and all $j \in P$; (3) $K$ (for $K \geq 2)$ is the cardinality restriction for cycles; and (4) $L$ (for $L \geq 3$ ) is the cardinality restriction for chains. The degree constraint $(10)$ is facet-defining for $\mathcal{Q}_{n, p, K, L}$ under the conditions that $|N| \geq 2$ and $|P| \geq 3$.

See Mak-Hau (2015b) for proofs of Theorems 3-5.

\section{FUTURE RESEARCH DIRECTIONS}

One promising direction for future research is to implement a Lagrangean relaxation-based branch-and-bound (BNB) method. For the CCMcP, one can solve an Assignment Problem (AP) relaxation at each node of the BNB, by relaxing Constraint (4), and dualising the violated constraints as a Lagrangean objective function. The AP can be solved in polynomial time. As the AP returns natural integer solutions, identifying cardinality violated cycles is straight forward. The Lagrangean Relaxation (LR) problem can be solved with a predetermined iterations of subgradient optimisation. This is likely to improve the quality of the upper bound with a reasonable computational effort.

With the CCCCP, at each node of the BNB tree, one can solve Model 2 with Constraints (14) and (15) relaxed, and explore the efficiency of computation and the quality of the upper bound from the relaxation. As optimisation problems defined on directed graphs that allow both cycles and chains have not been widely studied in the past, it is an interesting and challenging combinatorial optimisation problem in its own right. It would be beneficial to find stronger formulations and to explore hybrid exact methods, such as integer programming with various relaxation or decompositions, in combine with concepts of constraint programming and meta-heuristics.

\section{REFERENCES}

Abraham, D. J., A. Blum, and T. Sandholm (2007). Clearing algorithms for barter exchange markets: Enabling nationwide kidney exchanges. In Proceedings of the 8th ACM Conference on Electronic Commerce, EC '07, New York, NY, USA, pp. 295-304. ACM.

Anderson, R., I. Ashlagi, D. Gamarnik, and A. E. Roth (2015). Finding long chains in kidney exchange using the traveling salesman problem. Proceedings of the National Academy of Sciences.

Bai, G.-z. (2009, Dec). A new algorithm for k-cardinality assignment problem. In Computational Intelligence and Software Engineering, 2009. CiSE 2009. International Conference on, pp. 1-4.

Baldacci, R., P. Toth, and D. Vigo (2010). Exact algorithms for routing problems under vehicle capacity constraints. Annals of Operations Research 175(1), 213-245.

Bauer, P., J. Linderoth, and M. Savelsbergh (2002). A branch and cut approach to the cardinality constrained circuit problem. Mathematical Programming 91(2), 307-348. 
Cao, B. and F. Glover (1997, July). Tabu search and ejection chains\&mdash;application to a node weighted version of the cardinality-constrained tsp. Manage. Sci. 43(7), 908-921.

Constantino, M., X. Klimentova, A. Viana, and A. Rais (2013). New insights on integer-programming models for the kidney exchange problem. European Journal of Operational Research 231(1), 57 - 68.

Cornuejols, G. and F. Harche (1993, June). Polyhedral study of the capacitated vehicle routing problem. Math. Program. 60(1), 21-52.

Dell'Amico, M., A. Lodi, and S. Martello (2001). Efficient algorithms and codes for k-cardinality assignment problems. Discrete Applied Mathematics 110(1), 25 - 40. Proceedings of the First Conference on Algorithms and Experiments.

Dell'Amico, M. and S. Martello (1997). The k-cardinality assignment problem. Discrete Applied Mathematics 76(1-3), 103 - 121. Second International Colloquium on Graphs and Optimization.

Glorie, K. M., J. J. van de Klundert, and A. P. M. Wagelmans (2014). Kidney exchange with long chains: An efficient pricing algorithm for clearing barter exchanges with branch-and-price. Manufacturing \& Service Operations Management 16(4), 498-512.

Hartmann, M. and Özlük (2001). Facets of the p-cycle polytope. Discrete Applied Mathematics 112(13), 147 - 178. Combinatorial Optimization Symposium, Selected Papers.

Klimentova, X., F. Alvelos, and A. Viana (2014). A new branch-and-price approach for the kidney exchange problem. In B. Murgante and et al. (Eds.), Computational Science and Its Applications-ICCSA 2014, Volume 8580 of Lecture Notes in Computer Science, pp. 237-252. Springer International Publishing.

Mak, V. and N. Boland (2000). Heuristic approaches to the asymmetric travelling salesman problem with replenishment arcs. International Transactions in Operational Research 7(4-5), 431 - 447.

Mak, V. and N. Boland (2006). Facets of the polytope of the asymmetric travelling salesman problem with replenishment arcs. Discrete Optimization 3(1), 33 - 49. The Traveling Salesman Problem.

Mak, V. and N. Boland (2007). Polyhedral results and exact algorithms for the asymmetric travelling salesman problem with replenishment arcs. Discrete Applied Mathematics 155(16), 2093 - 2110.

Mak, V. and A. Ernst (2007). New cutting-planes for the time- and/or precedence-constrained ATSP and directed VRP. Mathematical Methods of Operations Research 66(1), 69-98.

Mak-Hau, V. (2015a). On the kidney exchange problem: cardinality constrained cycle and chain problems on directed graphs: a survey of integer programming approaches. Journal of Combinatorial Optimization, doi $=10.1007 / \mathrm{s} 10878-015-9932-4$.

Mak-Hau, V. (2015b). A polyhedral study of the cardinality constrained multi-cycle and multi-chain problem on directed graphs for kidney exchange optimization. http://wWw. deakin.edu.au/ vicky/ TechnicalReport $2 . p d f$

Manlove, D. and G. O'Malley (2012). Paired and altruistic kidney donation in the uk: Algorithms and experimentation. In R. Klasing (Ed.), Experimental Algorithms, Volume 7276 of Lecture Notes in Computer Science, pp. 271-282. Springer Berlin Heidelberg.

Nguyen, V. H. and J.-F. Maurras (2001). On the linear description of the k-cycle polytope. International Transactions in Operational Research 8(6), 673-692.

Patterson, R. and E. Rolland (2003, January). The cardinality constrained covering traveling salesman problem. Comput. Oper. Res. 30(1), 97-116.

Roth, A. E., T. Sünmez, and M. U. Ünver (2007). Efficient kidney exchange: Coincidence of wants in markets with compatibility-based preferences. American Economic Review 97(3), 828-851.

Toth, P. and D. Vigo (2002). Models, relaxations and exact approaches for the capacitated vehicle routing problem. Discrete Applied Mathematics 123(1-3), 487 - 512.

Volgenant, A. (2004). Solving the k-cardinality assignment problem by transformation. European Journal of Operational Research 157(2), $322-331$. 